\title{
LARGE AMPLITUDE FREE VIBRATION ANALYSIS OF TAPERED TIMOSHENKO BEAMS USING COUPLED DISPLACEMENT FIELD METHOD
}

\author{
K. RAJESH ${ }^{*}$ and K.M. SAHEB \\ Department of Mechanical Engineering, JNTUK \\ Kakinada, AP, INDIA-533 003 \\ E-mails: krajeshmech1112@gmail.com; meera.aec@gmail.com
}

\begin{abstract}
Tapered beams are more efficient compared to uniform beams as they provide a better distribution of mass and strength and also meet special functional requirements in many engineering applications. In this paper, the linear and non-linear fundamental frequency parameter values of the tapered Timoshenko beams are evaluated by using the coupled displacement field (CDF) method and closed form expressions are derived in terms of frequency ratio as a function of slenderness ratio, taper ratio and maximum amplitude ratio for hinged-hinged and clamped-clamped beam boundary conditions. The effectiveness of the CDF method is brought out through the solution of the large amplitude free vibrations, in terms of fundamental frequency of tapered Timoshenko beams with axially immovable ends. The results obtained by the present CDF method are validated with the existing literature wherever possible.
\end{abstract}

Key words: large amplitude vibrations, coupled displacement field method, tapered Timoshenko beams, taper ratio, slenderness ratio, frequency ratio.

\section{Introduction}

Research on vibrations of beams has been going on for a long period of time. So far, many authors have found different methods to find the free vibration behavior of shear flexible beams. Abrate [1] analyzed the free vibration of non-uniform beams with general shape and arbitrary boundary conditions. Byoung Koo Lee et al. [2] studied free vibrations of tapered beams with general boundary condition which involves finding an ordinary differential governing equation of beams which can be solved by numerical methods and the natural frequencies are calculated by combining the Runge Kutta method and the determinant search method. De Rosa et al. [3] considered the dynamic behavior of beams with an linearly varying cross-section in which the equation of motion is solved in terms of Bessel functions, and the boundary conditions lead to the frequency equation which is a function of four flexibility coefficients. De Rosa et al. [4] calculated the natural vibration frequencies of tapered beams by using the Euler-Bernoulli beam theory in the presence of an arbitrary number of rotationally, axially and elastically flexible constraints and the dynamic analysis is performed by means of the so-called cell discretization method (CDM), according to which the beam is reduced to a set of rigid bars, linked together by elastic sections, where the bending stiffness and the distributed mass of the bars is concentrated. Clementi et al. [5] studied the frequency response curves of a non-uniform beam undergoing nonlinear oscillations by using the multiple time scale method in which the axial inertia is neglected, and so the equations of motion are statically condensed on the transversal displacement only.

Firouz-Abadi et al. [6] investigated the transverse free vibration of a class of variable-cross-section beams using the Wentzel, Kramers, Brillouin (WKB) approximation in which the governing equation of motion of the Euler-Bernoulli beam including axial force distribution is utilized to obtain a singular differential equation in terms of the natural frequency of vibration and a WKB expansion series is applied to find the solution. Zamorska [7] used Green's function method for the free vibration problem of non uniform

\footnotetext{
* To whom correspondence should be addressed
} 
Bernoulli-Euler beams to find Green's function of the fourth order differential operator, occurring at the beam's equation of motion and proposed the power series method. Jung Woo Lee [8] developed the transfermatrix method to determine solutions to the free vibration characteristics of a tapered Bernoulli-Euler beam in which the roots of the differential equation are determined by using the Frobenius method to obtain the power series solution for bending vibrations and examined the effect of various taper ratios on the eigen pairs of beams, in which the height of the cross section along the length is linearly reduced.

Raju et al. [9] analyzed large amplitude free vibrations of tapered beams using continuum and finite element methods. Mahmoud et al. [10] applied the differential transformation method (DTM) for free vibration analysis of beams with uniform and non-uniform cross sections. Meera Saheb et al. [11] conducted a free vibration analysis for uniform Timoshenko beams using the coupled displacement field method. Mehmet Cem Ece et al. [12] studied the vibrations of an isotropic beam which has a variable cross-section. In [12], the governing equation is reduced to an ordinary differential equation in the spatial coordinate system for a family of cross-section geometries with exponentially varying width. Minmao Liao and Hongzhi Zhong [13] carried out a non-linear vibration analysis by establishing equations of motion for taper Timoshenko beams. Mahmoud Bayat [14] introduced an analytical study on the vibration frequencies of tapered beams using an ancient Chinese method called the Max-Min Approach (MMA) and Homotopy Perturbation Method (HPM) to obtain natural frequency and corresponding displacement of tapered beams. Mohamed Hussien Taha and Samir Abohadima [15] used a mathematical model for vibrations of nonuniform flexural beams by presenting a mathematical model for free vibrations of non-uniform viscoelastic flexural beams. An analytical solution for the fourth order differential equation of beam vibration under appropriate boundary conditions is obtained by factorization and calculated mode shapes and damped natural frequencies for a wide range of beam characteristics. Lewandowski [16] obtained an equation of motion to study non-linear vibrations of beams by employing Hamilton's principle by neglecting inertia forces and applied the Ritz method with continuum solution for determining natural frequencies.

Ramazan et al. [17] presented a free vibration analysis of a beam based on the Timoshenko type with different boundary conditions. The solutions are obtained by the method of Lagrange multipliers in which the free vibration problem is posed as a constrained vibration problem. Rossi and Laura [18] determined the natural vibration frequencies of linearly tapered beams subjected to different combinations of edge supports by finite element algorithmic procedures. Si Yuan et al. [19] utilized the exact dynamic stiffness method for studying vibration of Bernoulli-Euler members, for the case of flexural free vibration of non-uniform Timoshenko beams with no uniformity of geometric and material properties. Kukla and Zamojska [20] applied Green's function method in the frequency analysis of a beam with a varying cross section. The beam carries an arbitrary number of attached discrete systems. The exact solution to the problem concerns a beam with quadratically a varying cross-section area. Hoseini et al [21] used the homotopy analysis method to obtain an accurate analytical solution for fundamental non-linear natural frequency and corresponding displacement of tapered beams. Zhou and Cheung [22] studied the vibration characteristics of tapered beams with a continuously varying rectangular cross-section for a truncated beam and arbitrary positive numbers for a sharp ended beam and obtained the eigen frequency equation by the Rayleigh-Ritz method and the effect of the location convergence is discussed.

The solution for the large amplitude free vibration problems using energy methods involves assuming suitable admissible functions for lateral displacement and the total rotation which leads to two coupled nonlinear differential equations in terms of lateral displacement and the total rotation. This can be achieved with less computational effort by the coupled displacement field method in which lateral displacement and total rotation are coupled through the static equilibrium equation of the shear flexible beam. This method leads to only one undetermined coefficient which can be easily solved using the principle of conservation of total energy (neglecting damping) to solve the problem.

\section{Methodology}

\subsection{Rayleigh-Ritz method}

In the Rayleigh-Ritz method the expressions for strain energy and kinetic energy are 


$$
\begin{aligned}
& U=\frac{E I}{2} \int_{0}^{L}\left(\frac{d \theta}{d x}\right)^{2} d x+\frac{k G A}{2} \int_{0}^{L}\left(\frac{d w}{d x}+\theta\right)^{2} d x \\
& T=\frac{\rho A \omega_{L}^{2}}{2} \int_{0}^{L} w^{2} d x+\frac{\rho I \omega_{L}^{2}}{2} \int_{0}^{L} \theta^{2} d x
\end{aligned}
$$

where $\mathrm{E}$ is Young's modulus and $I$ is the area moment of inertia, $w$ is the lateral displacement, $\theta$ is the total rotation, $k$ is the shear correction factor, $G$ is the shear modulus, $A$ is the area of cross section, $\rho$ is the mass density of the material of the beam, $\omega$ is the radiant frequency, $L$ is the length of the beam and $x$ is the axial coordinate. Suitable admissible functions satisfying mainly the kinetic boundary conditions (sometimes the admissible functions may satisfy some or all the natural boundary conditions and do not violate the variation principles) are assumed for $w$ and $\theta$ as

$$
\begin{aligned}
& w=\sum_{i=1}^{n} a_{i} f_{i}(x), \\
& \theta=\sum_{i=1}^{n} b_{i} f_{i}{ }^{\prime}(x) .
\end{aligned}
$$

We consider the equivalence of $\theta$ and $\frac{d w}{d x}$ of the beam problem where $a_{i}$ and $b_{i}$ are the 2 nd undetermined coefficients for the multi-term admissible functions given by the above equations. For the sake of simplicity and clarity, single term admissible functions for $\theta$ and $w$ with two undetermined coefficients are chosen and it has been shown that the single term admissible functions with trigonometric functions for various boundary conditions of the beam are found to be accurate for all practical purposes. The Lagrangian (U-T) is minimized with respect to the two undetermined coefficients $a$ and $b$ as

$$
\begin{aligned}
& \frac{\partial(U-T)}{\partial a}=0, \\
& \frac{\partial(U-T)}{\partial b}=0 .
\end{aligned}
$$

By solving the above equations, a quadratic equation for the frequency parameter can be obtained in the form of $L \lambda^{2}-M \lambda+N=0$ which has roots of $\frac{-M \pm \sqrt{M^{2}-4 L N}}{2 L}$ and by solving the above equation the fundamental frequency parameter can be obtained as $\lambda=\frac{\rho A \omega_{L}{ }^{2} L^{4}}{E I}$ and can be solved to obtain the fundamental frequency parameter of Timoshenko beams for various boundary conditions as a function of the slenderness ratio $(\beta=L / r)$ and taper ratio, where $r$ is the radius of gyration.

The Rayleigh-Ritz (R-R) method is explained in detail as follows for a tapered Timoshenko hingedhinged beam boundary condition. The equation for strain energy and kinetic energy for a tapered Timoshenko beam are given as 


$$
\begin{aligned}
& U=\frac{E}{2} \int_{0}^{L} I\left(\frac{d \theta}{d x}\right)^{2} d x+\frac{k G}{2} \int_{0}^{L} A\left(\frac{d w}{d x}+\theta\right)^{2} d x, \\
& T=\frac{\rho \omega_{L}}{2} \int_{0}^{L} A w^{2} d x+\frac{\rho \omega_{L}^{2}}{2} \int_{0}^{L} I \theta^{2} d x, \\
& A=A_{0}\left(1+\frac{\alpha x}{L}\right), I=I_{0}\left(1+\frac{\alpha x}{L}\right)^{3}, \alpha=\frac{\left(h_{L}-h_{0}\right)}{h_{L}}
\end{aligned}
$$

where $h_{L}, h_{o}$, are the height of the beam at the left end $=0$ and the right end $=L$ respectively as given in Fig. 1 , $A_{o}$ and $I_{o}$ are cross sectional area and area moment of inertia at the right side, $A$ is the area at any cross section, $I$ is the moment of inertia at any cross section, $\alpha$ is the taper ratio. The assumed total rotation and transverse displacement for hinged-hinged beam are respectively

$$
\begin{aligned}
& \theta=b \frac{\pi}{L} \cos \frac{\pi x}{L}, \\
& w=a \sin \frac{\pi x}{L} .
\end{aligned}
$$

Substituting Eqs (2.9), (2.10) and (2.11) in Eq.(2.7) and after simplification, we get

$$
U=\frac{b^{2} E I_{0} \pi^{2}}{2 L}\left[\left(0.5+0.0871 \alpha^{3}+0.4241 \alpha^{2}+0.75 \alpha\right)\right]+\frac{k G A_{0} L}{2}\left[\left(b+\frac{a \pi}{L}\right)^{2}(0.5+0.25 \alpha)\right] \text {. }
$$

Substituting Eqs (2.9),(2.10) and (2.11) in Eq.(2.8) and after simplification, we obtain

$$
\begin{aligned}
& T=\frac{\rho \omega_{L}{ }^{2} a^{2} A_{0} L}{2}(0.5+0.25 \alpha)+\frac{\rho \omega_{L}{ }^{2} b^{2} I_{0} L}{2}\left(0.5+0.1629 \alpha^{3}+0.5759 \alpha^{2}+0.75 \alpha\right), \\
& \frac{\partial(U-T)}{\partial a}=0 .
\end{aligned}
$$

Applying the Lagrangian and minimizing with respect to $a$ and after simplification the equation becomes as

$$
\begin{aligned}
& a\left(k G A_{0} \frac{\pi^{2}}{L}-\rho \omega_{L}^{2} A_{0} L\right)+b k G A_{0} \pi=0, \\
& \frac{\partial(U-T)}{\partial b}=0
\end{aligned}
$$

Applying the Lagrangian and minimizing with respect to $b^{\prime} b^{\prime}$ and after simplification the equation becomes 


$$
\begin{aligned}
& a k G A_{0} \pi+b\left[\frac{E I_{0} \pi^{2}}{L} \frac{\left(0.5+0.0871 \alpha^{2}+0.4241 \alpha^{3}+0.75 \alpha\right)}{(0.5+0.25 \alpha)}+\right. \\
& \left.+k G A_{0} L-\rho \omega_{L}{ }^{2} I_{0} L \frac{\left(0.5+0.1629 \alpha^{3}+0.5759 \alpha^{2}+0.75 \alpha\right)}{(0.5+0.25 \alpha)}\right]=0
\end{aligned}
$$

Solving Eqs (2.15) and (2.17) and after simplification the final equation gets the following form

$$
L \lambda^{2}-M \lambda+N=0
$$

which has roots of $\lambda=\frac{-M \pm \sqrt{M^{2}-4 L N}}{2 L}$

where

$$
L=\frac{6.76 \varphi}{\beta^{6}}, \quad M=\left(\frac{21.3841}{\beta^{4}} \varphi+\frac{66.71834}{\beta^{4}} \psi+\frac{2.1666}{\beta^{2}}\right), \quad N=\frac{210.970}{\beta^{2}} \psi,
$$

$\lambda=\frac{\left(\frac{21.3841}{\beta^{4}} \varphi+\frac{66.71834}{\beta^{4}} \psi+\frac{2.1666}{\beta^{2}}\right) \pm \sqrt{\left(\frac{21.3841}{\beta^{4}} \varphi+\frac{66.71834}{\beta^{4}} \psi+\frac{2.1666}{\beta^{2}}\right)^{2}-\frac{5706.6297}{\beta^{8}} \psi \varphi}}{\frac{13.52}{\beta^{6}} \varphi}$

where

$$
\psi=\left(\frac{0.5+0.0871 \alpha^{3}+0.4241 \alpha^{2}+0.75 \alpha}{0.5+0.25 \alpha}\right) \varphi=\left(\frac{0.5+0.1629 \alpha^{3}+0.5759 \alpha^{2}+0.75 \alpha}{0.5+0.25 \alpha}\right)
$$
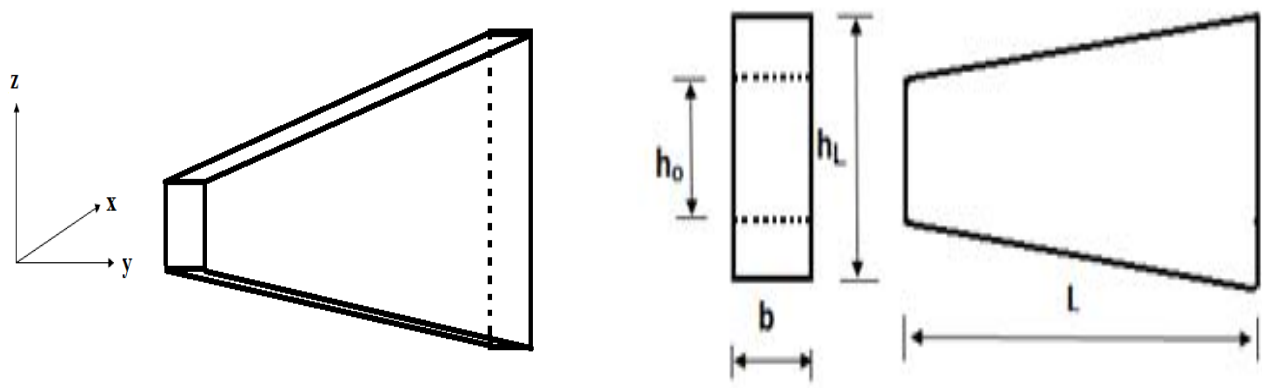

Fig.1. Tapered Timoshenko hinged-hinged beam with linearly varying height (constant width).

\subsection{Coupled displacement filed method}

Figure1 Tapered Timoshenko hinged-hinged beam with linearly varying height (constant width).

\subsubsection{Coupling equation}

From the kinematics of the shear flexible beam theory 


$$
\begin{aligned}
& \bar{u}=(x, z)=z(\theta), \\
& \bar{w}=(x, z)=w(x, z)
\end{aligned}
$$

where $\bar{u}$ is the axial displacement and $\bar{w}$ is the transverse displacements at any point of the beam, $z$ is the distance of any point from the neutral axis, $w$ is the transverse displacement and $\theta$ is the total rotation anywhere on the beam axis and $x, z$ are the independent spatial variables. The axial and shear strains are given by

$$
\begin{aligned}
& \in_{x}=z \frac{\partial \theta}{\partial x} \\
& \gamma_{x z}=\frac{\partial w}{\partial x}+\theta .
\end{aligned}
$$

Now, the expressions for the strain energy $U$ and the work done $W$ by the externally applied load are given by

$$
\begin{aligned}
& \mathrm{U}=\frac{E I}{2} \int_{O}^{L}\left(\frac{d \theta}{d x}\right)^{2} d x+\frac{k G A}{2} \int_{O}^{L}\left(\frac{\partial w}{\partial x}+\theta\right)^{2} d x, \\
& W=\int_{0}^{L} p(x) w(x) d x
\end{aligned}
$$

where $E I$ is the flexural rigidity, $G A$ is the shear rigidity, $\mathrm{k}$ is the shear correction factor (taken as $5 / 6$ in the present study), $p(x)$ is the static lateral load per unit length acting on the beam, $\mathrm{E}$ is Young's modulus, $G$ is the shear modulus, $x$ is the axial coordinate and $L$ is the length of the beam. Applying the principle of minimization of total potential energy, as

$$
\delta(U-W)=0
$$

the following equilibrium equations can be obtained

$$
\begin{gathered}
k G A\left(\frac{d^{2} w}{d x^{2}}+\frac{d \theta}{d x}\right)+p=0, \\
E I \frac{d^{2} \theta}{d x^{2}}-k G A\left(\frac{d w}{d x}+\theta\right)=0
\end{gathered}
$$

where $\theta$ is the total rotation, $w$ is the transverse displacement. Equations (2.27) and (2.28) are coupled equations and can be solved for obtaining the solution for the static analysis of the shear deformable beams.

A close observation of Eq.(2.27) shows that it is dependent on the load term ' $p$ ' and Eq.(2.28) is independent of the load term ' $p$ '. Hence, Eq.(2.28) is used to couple the total rotation $\theta$ and the transverse displacement $w$, so that the two undetermined coefficients problem (for single term admissible function) becomes a single undetermined coefficient problem and the resulting linear free vibration problem becomes much simpler to solve. 


\subsection{Evaluation of fundamental frequency parameter using coupled displacement field (CDF) method}

The concept of the coupled displacement field method is explained in detail in the following section. In the CDF method, the single term admissible function for the total rotation $(\theta)$ is assumed and the function for the transverse displacement $(w)$ is derived using the coupling equation. An admissible function for the total rotation $(\theta)$ is assumed for the tapered hinged-hinged beam which satisfies all the applicable boundary conditions and the symmetric condition in the beam domain.

$$
\begin{aligned}
& \theta=a \frac{\pi}{L} \cos \frac{\pi x}{L}, \\
& \frac{d \theta}{d x}=-a\left(\frac{\pi}{L}\right)^{2} \sin \frac{\pi x}{L}, \\
& \frac{d^{2} \theta}{d x^{2}}=-a \cos \frac{\pi x}{L} \frac{\pi^{3}}{L^{3}}
\end{aligned}
$$

where is the central lateral displacement of the beam which is also the maximum lateral displacement. Rewriting Eq.(2.28), we get

$$
\frac{d w}{d x}=-\theta+\frac{E I}{k G A} \frac{d^{2} \theta}{d x^{2}} .
$$

Substituting Eqs (2.29), (2.31) in Eq.(2.32) and by integrating the above equation, lateral displacement can be obtained as

$$
w=-a\left[1+\left(\frac{\pi}{L}\right)^{2} \gamma\right] \sin \pi \varsigma
$$

where

$$
\gamma=\frac{E I}{k G A}, \quad \varsigma=\frac{x}{L}
$$

It may be noted here that because of the coupled displacement field concept, the transverse displacement $w$ distribution contains the same undetermined coefficient $a$ as the $\theta$ distribution and satisfies all the applicable essential boundary and symmetric conditions.

$$
w(0)=w(L)=\left.\frac{d w}{d x}\right|_{x=L / 2}=0
$$

Linear free vibrations of tapered Timoshenko beams can be studied, once the coupled displacement field for the lateral displacement $w$, for an assumed $\theta$ distribution, is evaluated using the principle of conservation of total energy at any instant of time, neglecting damping, which states that $U+T=$ constant. The expression for $U$ and $T$ are already given in Eqs (2.7) and (2.8).

Substituting Eqs (2.9), (2.29), (2.30) and (2.32) in Eqs (2.7) and after simplification, we get 


$$
U=\frac{a^{2} E I_{0} \pi^{2}}{2 L}\left[\left(0.5+0.0871 \alpha^{3}+0.4241 \alpha^{2}+0.75 \alpha\right)+3.12 \frac{\pi^{2}}{\beta^{2}}(0.5+0.25 \alpha)\right]
$$

Substituting Eqs (2.9), (2.29) and (2.33) in Eq.(2.8) and after simplification, we obtain

$$
\begin{gathered}
T=\frac{a^{2} \rho A_{0} \omega_{L}^{2} L^{3}}{2 \pi^{2}}\left[\left(1+3.12 \frac{\pi^{2}}{\beta^{2}}\right)^{2}(0.5+0.25 \alpha)+\frac{\pi^{2}}{\beta^{2}}\left(0.5+0.1629 \alpha^{3}+0.5759 \alpha^{2}+0.75 \alpha\right)\right] \\
\frac{\partial(U-T)}{\partial a}=0
\end{gathered}
$$

by applying the Lagrangian, for Eqs (2.35), (2.36) the fundamental frequency parameter is obtained and is given as below

$$
\lambda=\frac{\rho A \omega_{L}^{2} L^{4}}{E I}=\frac{\pi^{4}\left[\left(0.5+0.0871 \alpha^{3}+0.4241 \alpha^{2}+0.75 \alpha\right)+3.12 \frac{\pi^{2}}{\beta^{2}}(0.5+0.25 \alpha)\right]}{\left[\left(1+3.12 \frac{\pi^{2}}{\beta^{2}}\right)^{2}(0.5+0.25 \alpha)+\frac{\pi^{2}}{\beta^{2}}\left(0.5+0.1629 \alpha^{3}+0.5759 \alpha^{2}+0.75 \alpha\right)\right]}
$$

Where $\lambda$ is the non dimensional fundamental frequency parameter, $\beta=L / r$ (slenderness ratio) and $r$ is the radius of gyration. The same procedure is adopted as discussed in the above section for calculating the fundamental frequency parameter for clamped-clamped tapered Timoshenko beam boundary condition (Tab.1).

\section{Large amplitude free vibrations}

For an assumed $\theta$ distribution, the coupled displacement field for the lateral displacement $w$ is evaluated, after the lateral displacement $w$ is calculated, the large amplitude free vibrations can be studied using the principle of conservation of total energy at any instant of time neglecting damping.

$$
U+T+W=\text { constant. }
$$

Work done due to large amplitudes

$$
W=\frac{T_{a}}{2} \int_{0}^{L} \frac{1}{2}\left(\frac{d w}{d x}\right)^{2} d x
$$

where $w$ is the transverse displacement obtained from the coupling equation

$$
T_{a}=\frac{E}{2 L r^{2}} \int_{0}^{L} I\left(\frac{d w}{d x}\right)^{2} d x
$$

It is to be noted here that $w$ in Eq.(3.3) does not contain shear flexible terms. $T_{a}$ is the tension developed in the beam because of large deformations. 
$W$ is the work done by the tension developed because of large amplitudes, $\rho$ is the mass density. Where $\mathrm{r}$ is the radius of gyration and $T_{a}$ is evaluated in terms of the amplitude ratio $(a / r)$. Substituting the values of $w$ (obtained from the coupled displacement field), i.e., Eq.(2.33) in Eq.(3.2) and solving the work done due to large amplitudes, we get

$$
W=\frac{E I_{0} \pi^{2} a^{4}}{16 r^{2} L} \frac{(2-\alpha)\left(\pi^{2} \alpha^{2}+3 \alpha^{2}-2 \pi^{2} \alpha+2 \pi^{2}\right)}{8 \pi^{2}}\left(1+\left(\frac{\pi}{L}\right)^{2} \frac{E I}{K G A}\right)^{2} .
$$

Substituting Eqs (2.35), (2.36) and (3.6) in Eq.(3.1) and simplifying.

The following form is obtained

$$
\dot{s}^{2}+s^{2} \alpha_{1}+s^{4} \alpha_{2}=\text { constant }
$$

where

$$
\begin{aligned}
& \alpha_{1}=\frac{E I_{0} 2 \pi^{4}}{\rho L^{4} A_{0}} \frac{\left[\left(0.5+0.0871 \alpha^{3}+0.4241 \alpha^{2}+0.75 \alpha\right)+\frac{3.12 \pi^{2}}{\beta^{2}}(0.5+0.25 \alpha)\right]}{\left[\left(1+\frac{3.12 \pi^{2}}{\beta^{2}}\right)^{2}(0.5+0.25 \alpha)+\frac{\pi^{2}}{\beta^{2}}\left(0.5+0.1629 \alpha^{3}+0.5759 \alpha^{2}+0.75 \alpha\right)\right]}, \\
& \alpha_{2}=\frac{3 E I_{0} \pi^{2}}{35 \rho L^{4} A_{0} r^{2}} \frac{\left[(2-\alpha)\left(\pi^{2} \alpha^{2}+3 \alpha^{2}-2 \pi^{2} \alpha+2 \pi^{2}\right)\left(1+\frac{3.12 \pi^{2}}{\beta^{2}}\right)^{2}\right]}{\left[\left(1+\frac{3.12 \pi^{2}}{\beta^{2}}\right)^{2}(0.5+0.25 \alpha)+\frac{\pi^{2}}{\beta^{2}}\left(0.5+0.1629 \alpha^{3}+0.5759 \alpha^{2}+0.75 \alpha\right)\right]}
\end{aligned}
$$

The ratio of non linear and linear frequency is expressed as

$$
\begin{aligned}
& \left(\frac{\omega_{N L}}{\omega_{L}}\right)^{2}=1+\frac{3}{2}\left(\frac{\alpha_{2}}{\alpha_{1}}\right)\left(\frac{a_{m}}{r}\right)^{2}, \\
& \left(\frac{\omega_{N L}}{\omega_{L}}\right)^{2}=1+\frac{3}{70} \frac{(2-\alpha)\left(\pi^{2} \alpha^{2}+3 \alpha^{2}-2 \pi^{2} \alpha+2 \pi^{2}\right)\left(1+\left(\frac{3.12 \pi^{2}}{\beta^{2}}\right)\right)^{2}}{\pi^{2}\left[\left(0.5+0.0871 \alpha^{3}+0.4241 \alpha^{2}+0.75 \alpha\right)+\frac{3.12 \pi^{2}}{\beta^{2}}(0.5+0.25 \alpha)\right]}\left(\frac{a_{m}}{r}\right)^{2} .
\end{aligned}
$$

The same procedure is adopted as in the above section for calculating the frequency ratio for clampedclamped tapered Timoshenko beam boundary condition; respective important expressions are given in Tab.1.

\section{Numerical results and discussion}

In all computations, Poisson's ratio (v) and shear correction factor $(k)$ are taken as 0.3 and $5 / 6$ respectively. The concept of the coupled displacement field is used to determine the ratios of non-linear radian frequency $\omega_{N L}$ to the linear radian frequency $\omega_{L}$ of tapered Timoshenko beams with the two most practically used hinged-hinged, clamped-clamped beam boundary conditions. The boundary conditions of 
the beam considered here are hinged-hinged, clamped-clamped with axially immovable ends. Suitable single term trigonometric admissible functions are used to represent the total rotation $\theta$ in the coupled displacement field method. The corresponding coupled lateral displacement $\mathrm{w}$ is derived using the coupling equation. The numerical results are obtained in terms of $\left(\frac{\omega_{N L}}{\omega_{L}}\right)$ for various maximum amplitudes, taper parameters and slenderness ratios. To assess the accuracy of the results, the present results obtained from the coupled displacement field method are compared with the existing literature.

Table 1 shows the expressions for the total rotation $(\theta)$, derived transverse displacement $(w)$, fundamental frequency parameter $(\lambda)$ and frequency ratio $\left(\frac{\omega_{N L}}{\omega_{L}}\right)$ for a clamped-clamped tapered Timoshenko beam. Tables 2 and 3 shows the variation of linear non-dimensional fundamental frequency parameter as a function of the slenderness ratio and taper ratio for hinged-hinged, clamped-clamped beam boundary conditions. To show the effectiveness of the CDF method, the author also solved the tapered Timoshenko hinged-hinged beam boundary condition problem using the famous conventional Rayleigh-Ritz method and the same results are also included in Tab.3 along with the results of other researchers wherever possible. It is observed from Tab.2 and Tab.3 that the non dimensional linear fundamental frequency parameter value increases with an increase in the taper ratio for a given slenderness ratio. It is also found from Tab.2 and Tab.3 that the non dimensional linear fundamental frequency parameter value increases with an increase in the slenderness ratio for a given taper parameter. It is in general observed from Tab.2 and Tab.3 that more frequency values are observed in the case of clamped-clamped beam when compared to the hinged-hinged beam.

Table.1. Expressions for total rotation $(\theta)$, derived transverse displacement $(w)$ fundamental frequency parameter $(\lambda)$ and frequency ratio $\left(\frac{\omega_{N L}}{\omega_{L}}\right)^{2}$ for clamped-clamped tapered Timoshenko beams.

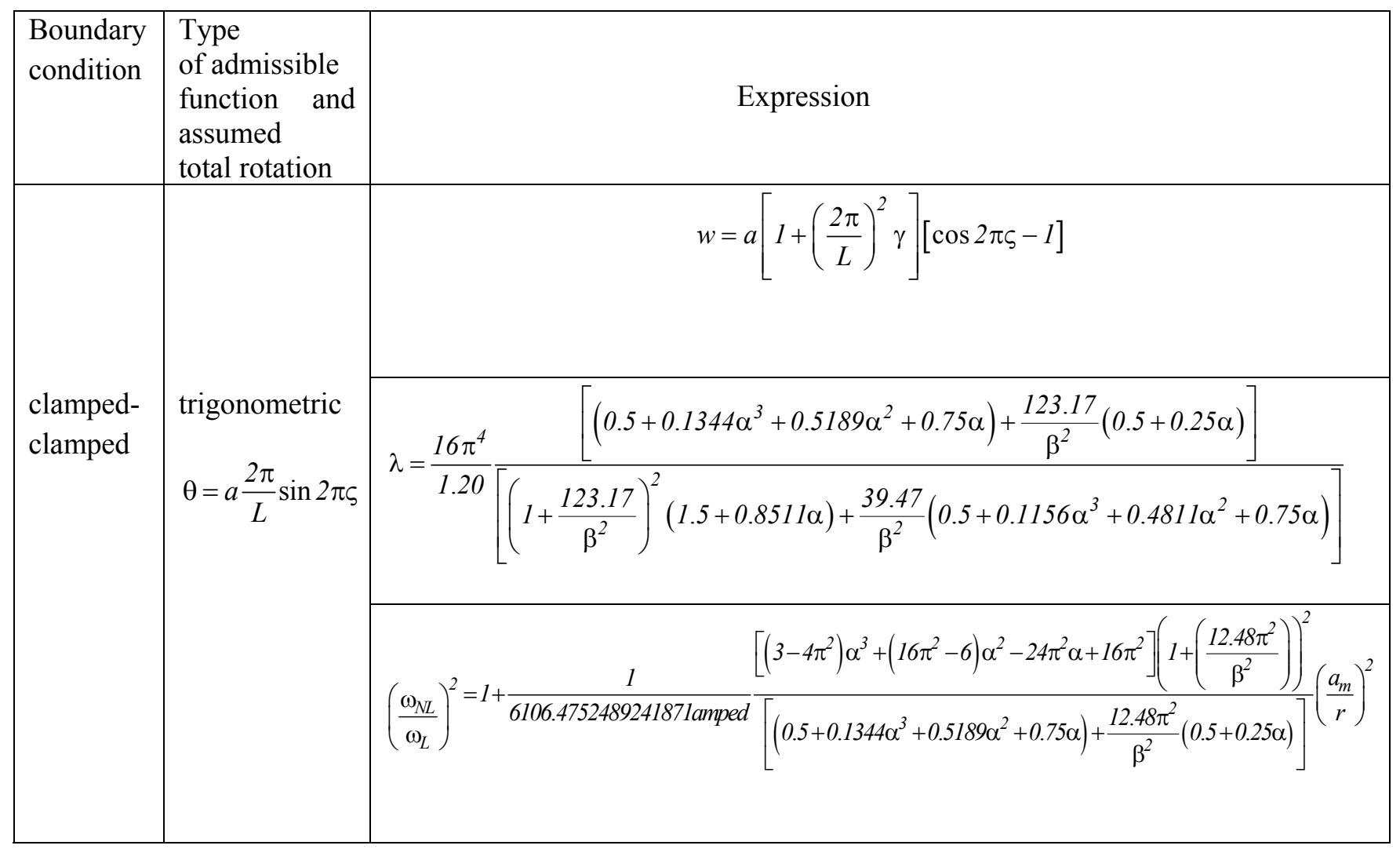


Table 2. $\lambda^{1 / 2}$ values for a tapered Timoshenko hinged-hinged beam.

\begin{tabular}{|c|c|c|c|c|c|c|c|c|c|c|c|c|}
\hline \multirow{3}{*}{\begin{tabular}{|c|} 
\\
Taper \\
ratio \\
$(\alpha)$ \\
\end{tabular}} & \multicolumn{12}{|c|}{ Slenderness ratio $(\beta)$} \\
\hline & \multicolumn{3}{|c|}{10} & \multicolumn{3}{|c|}{20} & \multirow{2}{*}{$\begin{array}{c}23.0951 \\
\text { CDF } \\
\text { Method }\end{array}$} & \multicolumn{2}{|c|}{40} & \multicolumn{3}{|c|}{100} \\
\hline & $\begin{array}{c}\text { CDF } \\
\text { Method }\end{array}$ & $\begin{array}{c}\text { R-R } \\
\text { method }\end{array}$ & Ref[18] & $\begin{array}{c}\text { CDF } \\
\text { Method }\end{array}$ & $\begin{array}{c}\text { R-R } \\
\text { method }\end{array}$ & $\operatorname{Ref}[18]$ & & $\begin{array}{r}\text { CDF } \\
\text { Method }\end{array}$ & $\begin{array}{c}\text { R-R } \\
\text { Method }\end{array}$ & $\begin{array}{c}\text { CDF } \\
\text { Method }\end{array}$ & $\begin{array}{c}\text { R-R } \\
\text { Method }\end{array}$ & Ref[18] \\
\hline 0 & 8.3912 & 8.6913 & 8.388 & 9.4107 & 9.8398 & 9.411 & $\begin{array}{r}9.5180 \\
9.5163^{\wedge} \\
9.5163^{\S}\end{array}$ & 9.7470 & 10.2259 & 9.8496 & 10.3445 & 9.850 \\
\hline 0.15 & 8.8435 & 8.8404 & 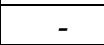 & 10.0595 & 10.0547 & - & 9.96419 & 10.4695 & 10.4676 & 10.5953 & 10.5950 & - \\
\hline 0.1 & 8.6916 & 8.9874 & 8.683 & 9.8415 & 9.2696 & 9.829 & 10.1899 & 10.2267 & 10.7107 & 10.3446 & 10.8473 & - \\
\hline 0.2 & 8.9962 & 9.1322 & 8.955 & 10.2789 & 10.4843 & 10.228 & 10.4171 & 10.7141 & 10.9549 & 10.8480 & 11.1014 & - \\
\hline 0.25 & 9.1496 & 9.2747 & 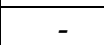 & 10.4996 & 10.6987 & - & 10.6458 & 10.9604 & 11.2002 & 11.1024 & 11.3570 & - \\
\hline 0.3 & 9.3036 & 9.4148 & 9.205 & 10.7214 & 10.9125 & 10.610 & 10.8757 & 11.2082 & 11.4463 & 11.3585 & 11.6141 & - \\
\hline 0.35 & 9.4580 & 9.5526 & 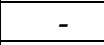 & 10.9443 & 11.1257 & 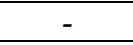 & 11.1068 & 11.4574 & 11.6933 & 11.6161 & 11.8726 & - \\
\hline 0.4 & 9.6127 & 9.6880 & - & 11.1681 & 11.3382 & - & 11.3389 & 11.7079 & 11.9409 & 11.8752 & 12.1323 & - \\
\hline 0.45 & 9.7676 & 9.8209 & - & 11.3926 & 11.5498 & - & 11.5718 & 11.9596 & 12.1891 & 12.1356 & 12.3932 & - \\
\hline 0.5 & 9.9225 & 9.9514 & - & 11.6178 & 11.7604 & - & 11.8055 & 12.2124 & 12.4378 & 12.3973 & 12.6551 & - \\
\hline 0.55 & 10.0774 & 10.0795 & - & 11.8435 & 11.9700 & - & 12.0400 & 12.4661 & 12.6869 & 12.6601 & 12.9181 & - \\
\hline 0.6 & 10.2321 & 10.2051 & - & 12.0697 & 12.1785 & 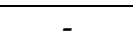 & 12.2749 & 12.7208 & 12.9362 & 12.9241 & 13.1820 & - \\
\hline 0.65 & 10.3866 & 10.3283 & - & 12.2962 & 12.3857 & - & 12.5104 & 12.9764 & 13.1858 & 13.1891 & 13.4468 & - \\
\hline 0.7 & 10.5407 & 10.4491 & - & 12.5230 & 12.5917 & - & 12.7463 & 13.2327 & 13.4355 & 13.4551 & 13.7123 & - \\
\hline 0.75 & 10.6943 & 10.5675 & - & & 12.7963 & - & 25 & 13.4897 & 13.6 & 13.7219 & 13.9787 & - \\
\hline 0.8 & 10.8475 & 10.6835 & - & 12.9771 & 12.9995 & - & 13.2190 & 13.7474 & 13.9351 & 13.9897 & 14.2457 & - \\
\hline 0.85 & 11.0000 & 10.7971 & - & 13.2042 & 13.2013 & - & 13.4557 & 14.0057 & 14.1848 & 14.2582 & 14.5133 & - \\
\hline 0.9 & 11.1519 & 10.9084 & - & 13.4313 & 13.4016 & - & 13.6924 & 14.2644 & 14.4345 & 14.5274 & 14.7816 & - \\
\hline 0.95 & 11.3030 & 11.0175 & - & 13.6583 & 13.6003 & - & 13.9293 & 14.5237 & 14.6840 & 14.7974 & 15.0504 & - \\
\hline 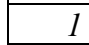 & 11.4533 & 11.3873 & - & 13.8852 & 13.4105 & - & 14.1661 & 14.7834 & 14.7470 & 15.0681 & 15.8495 & - \\
\hline
\end{tabular}

Table 3. $\lambda^{1 / 2}$ values for a tapered Timoshenko clamped-clamped beam.

\begin{tabular}{|c|c|c|c|c|c|c|c|c|}
\hline \multirow[b]{3}{*}{$\begin{array}{l}\text { Taper Ratio } \\
(\alpha)\end{array}$} & \multicolumn{8}{|c|}{ Slenderness ratio $(\beta)$} \\
\hline & \multicolumn{2}{|c|}{10} & \multicolumn{2}{|c|}{20} & \multirow{2}{*}{$\begin{array}{c}40 \\
\text { CDF } \\
\text { method }\end{array}$} & \multirow{2}{*}{$\begin{array}{c}80 \\
\begin{array}{c}\text { CDF } \\
\text { method }\end{array}\end{array}$} & \multicolumn{2}{|c|}{100} \\
\hline & $\begin{array}{c}\text { CDF } \\
\text { Method }\end{array}$ & $\operatorname{Ref}[18]$ & $\begin{array}{c}\text { CDF } \\
\text { Method }\end{array}$ & $\operatorname{Ref[18]}$ & & & $\begin{array}{c}\text { CDF } \\
\text { Method }\end{array}$ & Ref[18] \\
\hline 0 & 13.8025 & 13.8370 & 18.0930 & 18.838 & 21.8857 & 22.5543 & 22.6392 & 22.61 \\
\hline 0.1 & 14.0619 & 14.0910 & 18.7304 & 19.487 & 22.8503 & 23.6136 & 23.7109 & - \\
\hline 0.15 & 14.2010 & - & 19.0651 & - & 23.3531 & 24.1647 & 24.2683 & - \\
\hline 0.2 & 14.3459 & 14.3180 & 19.4092 & 20.095 & 23.8682 & 24.7287 & 24.8387 & - \\
\hline 0.25 & 14.4964 & - & 19.7623 & - & 24.3947 & 25.3046 & 25.4210 & - \\
\hline 0.3 & 14.6522 & 14.5210 & 20.1240 & 20.667 & 24.9316 & 25.8914 & 26.0144 & - \\
\hline 0.35 & 14.8130 & - & 20.4928 & - & 25.4783 & 26.4884 & 26.6180 & - \\
\hline 0.4 & 14.9785 & - & 20.8690 & - & 26.0340 & 27.0948 & 27.2310 & - \\
\hline 0.45 & 15.1484 & - & 21.2519 & - & 26.5980 & 27.7099 & 27.8528 & - \\
\hline 0.5 & 15.3226 & - & 21.6408 & - & 27.1696 & 28.3331 & 28.4827 & - \\
\hline 0.55 & 15.5008 & - & 22.0355 & - & 27.7484 & 28.9638 & 29.1202 & - \\
\hline 0.6 & 15.6828 & - & 22.4354 & - & 28.3338 & 29.6014 & 29.7646 & - \\
\hline 0.65 & 15.8683 & - & 22.8401 & - & 28.9252 & 30.2454 & 30.4156 & - \\
\hline 0.7 & 16.0572 & - & 23.2493 & - & 29.5223 & 30.8955 & 31.0726 & - \\
\hline 0.75 & 16.2492 & - & 23.6626 & - & 30.1246 & 31.5511 & 31.7352 & - \\
\hline 0.8 & 16.4441 & - & 24.0797 & - & 30.7317 & 32.2119 & 32.4030 & - \\
\hline 0.85 & 16.6418 & - & 24.5002 & - & 31.3433 & 32.8776 & 33.0758 & - \\
\hline 0.9 & 16.8421 & - & 24.9240 & - & 31.9591 & 33.5477 & 33.7531 & - \\
\hline 0.95 & 17.0448 & - & 25.3560 & - & 32.5786 & 34.2220 & 34.4346 & - \\
\hline 1 & 17.2498 & - & 25.7799 & - & 33.2017 & 34.9003 & 35.1201 & - \\
\hline
\end{tabular}


In Tabs 4, 5, 6 and 7 the variation of the frequency ratio $\left(\frac{\omega_{N L}}{\omega_{L}}\right)$ with the maximum amplitude ratio and taper parameters for different slenderness ratios such as 20 (short beams) and 100 (slender beams). They are given respectively for hinged-hinged, clamped-clamped tapered beam boundary conditions. It is found from Tab.4 and Tab.5 that the frequency ratio is a function of three parameters such as the maximum amplitude ratio, taper parameter and slenderness ratio. It is in general found from Tab.4 and Tab.5 that the frequency ratio increases with an increase of the maximum amplitude ratio for a given taper parameter and slenderness ratio. It is also seen in Tab.4 and Tab.5 that the frequency ratio decreases with an increase of the taper parameter for a given slenderness ratio and amplitude ratio. This is mainly due to the fact that as the taper parameter increases the mass of the beam decreases. A similar frequency ratio variation has been observed for clamped-clamped beam boundary conditions for the slenderness ratio of 20 and 100 and are included in Tab. 6 and Tab.7

Table 4. $\frac{\omega_{N L}}{\omega_{L}}$ for a tapered shear flexible hinged-hinged beam for $\beta=20$.

\begin{tabular}{|l|c|c|c|c|c|c|c|c|}
\hline & \multicolumn{2}{|c|}{$\alpha=0.25$} & \multicolumn{2}{c|}{$\alpha=0.5$} & \multicolumn{2}{c|}{$\alpha=0.75$} & \multicolumn{2}{c|}{$\alpha=1$} \\
\cline { 2 - 9 }$a_{m} / r$ & $\begin{array}{c}\text { CDF } \\
\text { Method }\end{array}$ & Ref [13] & $\begin{array}{c}\text { CDF } \\
\text { Method }\end{array}$ & Ref [13] & $\begin{array}{c}\text { CDF } \\
\text { Method }\end{array}$ & $\begin{array}{c}\text { CDF }[13] \\
\text { Method }\end{array}$ & Ref [13] \\
\hline 0.10 & 1.0009 & 1.0009 & 1.0005 & 1.0007 & 1.0003 & 1.0006 & 1.0002 & 1.0005 \\
\hline 0.20 & 1.0036 & 1.0037 & 1.0019 & 1.0030 & 1.0011 & 1.0025 & 1.0007 & 1.0021 \\
\hline 0.30 & 1.0081 & - & 1.0042 & - & 1.0025 & - & 1.0016 & - \\
\hline 0.40 & 1.0144 & 1.0146 & 1.0075 & 1.0119 & 1.0044 & 1.0100 & 1.0028 & 1.0085 \\
\hline 0.50 & 1.0224 & - & 1.0118 & - & 1.0069 & - & 1.0044 & - \\
\hline 0.60 & 1.0321 & 1.0325 & 1.0169 & 1.0266 & 1.0099 & 1.0224 & 1.0064 & 1.0190 \\
\hline 0.70 & 1.0434 & - & 1.0230 & - & 1.0134 & - & 1.0087 & - \\
\hline 0.80 & 1.0564 & 1.0570 & 1.0299 & 1.0467 & 1.0175 & 1.0394 & 1.0113 & 1.0336 \\
\hline 0.90 & 1.0709 & - & 1.0377 & - & 1.0221 & - & 1.0143 & - \\
\hline 1.00 & 1.0868 & 1.0878 & 1.0464 & 1.0721 & 1.0272 & 1.0608 & 1.0177 & 1.0519 \\
\hline 1.10 & 1.1042 & - & 1.0559 & - & 1.0328 & - & 1.0213 & - \\
\hline 1.20 & 1.1230 & 1.1239 & 1.0662 & 1.1022 & 1.0389 & 1.0864 & 1.0253 & 1.0740 \\
\hline 1.30 & 1.1430 & - & 1.0773 & - & 1.0455 & - & 1.0297 & - \\
\hline 1.40 & 1.1642 & - & 1.0891 & - & 1.0526 & - & 1.0343 & - \\
\hline 1.50 & 1.1865 & 1.1878 & 1.1017 & 1.1552 & 1.0602 & 1.1315 & 1.0393 & 1.1131 \\
\hline 2 & 1.3135 & & 1.1748 & & 1.1047 & & 1.0689 & \\
\hline 3 & 1.6224 & & 1.3622 & & 1.2231 & & 1.1492 & \\
\hline 4 & 1.9752 & & 1.5878 & & 1.3718 & & 1.2531 & \\
\hline 5 & 2.3523 & & 1.8376 & & 1.5420 & & 1.3751 & \\
\hline
\end{tabular}


Table 5. $\frac{\omega_{N L}}{\omega_{L}}$ for a tapered shear flexible hinged-hinged beam for $\beta=100$. $\omega_{L}$

\begin{tabular}{|c|c|c|c|c|c|c|c|c|c|c|}
\hline & \multicolumn{10}{|c|}{$\alpha$} \\
\hline & \multicolumn{2}{|c|}{0.25} & \multicolumn{2}{|c|}{0.4} & \multicolumn{2}{c|}{0.5} & \multicolumn{2}{c|}{0.75} & \multicolumn{2}{|c|}{1} \\
\cline { 2 - 13 }$a_{m} / r$ & $\begin{array}{c}\text { CDF } \\
\text { Method }\end{array}$ & Ref[13] & $\begin{array}{c}\text { CDF } \\
\text { Method }\end{array}$ & Ref[9] & $\begin{array}{l}\text { CDF } \\
\text { Method }\end{array}$ & Ref[13] & $\begin{array}{l}\text { CDF } \\
\text { Method }\end{array}$ & Ref[13] & $\begin{array}{l}\text { CDF } \\
\text { Method }\end{array}$ & Ref[13] \\
\hline 0.10 & 1.0008 & 1.0010 & 1.0010 & 1.0010 & 1.0004 & 1.0008 & 1.0003 & 1.0007 & 1.0002 & 1.0006 \\
\hline 0.20 & 1.0033 & 1.0040 & 1.0022 & 1.0042 & 1.0017 & 1.0033 & 1.0010 & 1.0028 & 1.0006 & 1.0025 \\
\hline 0.30 & 1.0075 & - & 1.0050 & - & 1.0039 & - & 1.0022 & - & 1.0014 & - \\
\hline 0.40 & 1.0132 & 1.0158 & 1.0088 & 1.0166 & 1.0069 & 1.0132 & 1.0040 & 1.0113 & 1.0025 & 1.0098 \\
\hline 0.50 & 1.0206 & - & 1.0138 & - & 1.0107 & - & 1.0062 & - & 1.0040 & - \\
\hline 0.60 & 1.0295 & 1.0353 & 1.0198 & 1.0370 & 1.0154 & 1.0294 & 1.0089 & 1.0252 & 1.0057 & 1.0219 \\
\hline 0.70 & 1.0400 & - & 1.0268 & - & 1.0209 & - & 1.0121 & - & 1.0078 & - \\
\hline 0.80 & 1.0519 & 1.0619 & 1.0349 & 1.0649 & 1.0272 & 1.0516 & 1.0158 & 1.0444 & 1.0102 & 1.0387 \\
\hline 0.90 & 1.0653 & - & 1.0440 & - & 1.0344 & - & 1.0199 & - & 1.0128 & - \\
\hline 1.00 & 1.0800 & 1.0950 & 1.0541 & 1.0997 & 1.0423 & 1.0795 & 1.0245 & 1.0685 & 1.0158 & 1.0597 \\
\hline 1.10 & 1.0961 & - & 1.0651 & - & 1.0509 & - & 1.0296 & - & 1.0191 & - \\
\hline 1.20 & 1.1134 & 1.1344 & 1.0770 & - & 1.0603 & 1.1127 & 1.0351 & 1.0972 & 1.0227 & 1.0849 \\
\hline 1.30 & 1.1319 & - & 1.0898 & - & 1.0704 & - & 1.0411 & - & 1.0266 & - \\
\hline 1.40 & 1.1516 & - & 1.1035 & - & 1.0813 & - & 1.0475 & - & 1.0308 & - \\
\hline 1.50 & 1.1724 & 1.2033 & 1.1180 & - & 1.0928 & 1.1712 & 1.0543 & 1.1479 & 1.0352 & 1.1296 \\
\hline 2 & 1.2906 & & 1.2017 & 1.3354 & 1.1598 & & 1.0948 & & 1.0619 & \\
\hline 3 & 1.5805 & & 1.4140 & 1.6981 & 1.3330 & & 1.2027 & & 1.1344 & \\
\hline 4 & 1.9138 & & 1.6663 & - & 1.5430 & & 1.3394 & & 1.2289 & \\
\hline 5 & 2.2717 & & 1.9432 & - & 1.7769 & & 1.4968 & & 1.3406 & \\
\hline
\end{tabular}

Table 6. $\frac{\omega_{N L}}{\omega_{L}}$ for a tapered shear flexible clamped-clamped beam for $\beta=20$.

\begin{tabular}{|c|c|c|c|c|c|c|c|c|}
\hline \multirow[b]{3}{*}{$a_{m} / r$} & \multicolumn{8}{|c|}{$\alpha$} \\
\hline & \multicolumn{2}{|c|}{0.25} & \multicolumn{2}{|c|}{0.5} & \multicolumn{2}{|c|}{0.75} & \multicolumn{2}{|c|}{1} \\
\hline & $\begin{array}{c}\text { CDF } \\
\text { Method }\end{array}$ & Ref 13] & $\begin{array}{c}\text { CDF } \\
\text { Method }\end{array}$ & Ref[13] & $\begin{array}{c}\text { CDF } \\
\text { Method }\end{array}$ & Ref 13] & $\begin{array}{c}\text { CDF } \\
\text { Method }\end{array}$ & Ref[13] \\
\hline 0.10 & 1.0002 & 1.0002 & 1.0001 & 1.0001 & 1.0000 & 1.0001 & 1.0000 & 1.0001 \\
\hline 0.20 & 1.0006 & 1.0006 & 1.0003 & 1.0005 & 1.0002 & 1.0004 & 1.0001 & 1.0003 \\
\hline 0.30 & 1.0016 & - & 1.0008 & - & 1.0004 & - & 1.0002 & - \\
\hline 0.40 & 1.0028 & 1.0025 & 1.0014 & 1.0019 & 1.0007 & 1.0015 & 1.0004 & 1.0012 \\
\hline 0.50 & 1.0043 & - & 1.0022 & - & 1.0011 & - & 1.0006 & - \\
\hline 0.60 & 1.0062 & 1.0057 & 1.0031 & 1.0043 & 1.0016 & 1.0034 & 1.0009 & 1.0026 \\
\hline 0.70 & 1.0084 & 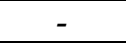 & 1.0043 & - & 1.0023 & - & 1.0012 & - \\
\hline 0.80 & 1.0110 & 1.0100 & 1.0056 & 1.0076 & 1.0029 & 1.0060 & 1.0015 & 1.0047 \\
\hline 0.90 & 1.0139 & - & 1.0070 & - & 1.0036 & - & 1.0019 & - \\
\hline 1.00 & 1.0172 & 1.0156 & 1.0087 & 1.0119 & 1.0045 & 1.0093 & 1.0024 & 1.0073 \\
\hline 1.10 & 1.0207 & - & 1.0105 & - & 1.0054 & - & 1.0029 & - \\
\hline 1.20 & 1.0246 & 1.0223 & 1.0125 & 1.0170 & 1.0065 & 1.0133 & 1.0034 & 1.0105 \\
\hline 1.30 & 1.0288 & - & 1.0146 & - & 1.0076 & - & 1.0040 & - \\
\hline 1.40 & 1.0334 & - & 1.0169 & 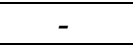 & 1.0088 & - & 1.0047 & - \\
\hline 1.50 & 1.0382 & 1.0344 & 1.0194 & 1.0263 & 1.0100 & 1.0206 & 1.0054 & 1.0164 \\
\hline 2 & 1.0670 & & 1.0343 & & 1.0178 & & 1.0095 & \\
\hline 3 & 1.1452 & & 1.0756 & & 1.0397 & & 1.0213 & \\
\hline 4 & 1.2464 & & 1.1308 & & 1.0694 & & 1.0376 & \\
\hline 5 & 1.3657 & & 1.1982 & & 1.1066 & & 1.0582 & \\
\hline
\end{tabular}


Table 7. $\frac{\omega_{N L}}{\omega_{L}}$ for a tapered shear flexible clamped-clamped beam for $\beta=100$.

\begin{tabular}{|c|c|c|c|c|c|c|c|c|c|c|}
\hline \multirow[b]{3}{*}{$a_{m} / r$} & \multicolumn{10}{|c|}{$\alpha$} \\
\hline & \multicolumn{2}{|c|}{0.25} & \multicolumn{2}{|c|}{0.4} & \multicolumn{2}{|c|}{0.5} & \multicolumn{2}{|c|}{0.75} & \multicolumn{2}{|c|}{1} \\
\hline & $\begin{array}{c}\text { CDF } \\
\text { Method }\end{array}$ & $\begin{array}{l}\text { Ref } \\
{[13]}\end{array}$ & $\begin{array}{c}\text { CDF } \\
\text { Method }\end{array}$ & $\operatorname{Ref}[9]$ & $\begin{array}{l}\text { CDF } \\
\text { Method }\end{array}$ & $\operatorname{Ref}[13]$ & $\begin{array}{l}\text { CDF } \\
\text { Method }\end{array}$ & $\operatorname{Ref[13]~}$ & $\begin{array}{l}\text { CDF } \\
\text { Method }\end{array}$ & Ref[13] \\
\hline 0.10 & 1.0001 & 1.0002 & 1.0001 & 1.0004 & 1.0001 & 1.0002 & 1.0000 & 1.0002 & 1.0000 & 1.0001 \\
\hline 0.20 & 1.0005 & 1.0009 & 1.0003 & 1.0017 & 1.0003 & 1.0008 & 1.0001 & 1.0007 & 1.0001 & 1.0006 \\
\hline 0.30 & 1.0011 & - & 1.0007 & - & 1.0006 & - & 1.0003 & - & 1.0001 & - \\
\hline 0.40 & 1.0020 & 1.0038 & 1.0013 & 1.0066 & 1.0010 & 1.0031 & 1.0005 & 1.0027 & 1.0003 & 1.0023 \\
\hline 0.50 & 1.0032 & - & 1.0021 & - & 1.0015 & - & 1.0008 & - & 1.0004 & - \\
\hline 0.60 & 1.0046 & 1.0085 & 1.0030 & 1.0149 & 1.0022 & 1.0070 & 1.0011 & 1.0060 & 1.0006 & 1.0052 \\
\hline 0.70 & 1.0062 & - & 1.0040 & - & 1.0030 & - & 1.0015 & - & 1.0008 & - \\
\hline 0.80 & 1.0081 & 1.0150 & 1.0052 & 1.0263 & 1.0039 & 1.0124 & 1.0020 & 1.0106 & 1.0010 & 1.0092 \\
\hline 0.90 & 1.0103 & - & 1.0066 & - & 1.0050 & - & 1.0025 & - & 1.0013 & - \\
\hline 1.00 & 1.0126 & 1.0233 & 1.0082 & 1.0408 & 1.0061 & 1.0193 & 1.0030 & 1.0165 & 1.0016 & 1.0144 \\
\hline 1.10 & 1.0153 & - & 1.0099 & & 1.0074 & - & 1.0037 & - & 1.0019 & - \\
\hline 1.20 & 1.0182 & 1.0334 & 1.0118 & & 1.0088 & 1.0278 & 1.0044 & 1.0237 & 1.0023 & 1.0206 \\
\hline 1.30 & 1.0213 & - & 1.0138 & & 1.0103 & - & 1.0052 & - & 1.0027 & - \\
\hline 1.40 & 1.0246 & - & 1.0160 & & 1.0120 & - & 1.0060 & - & 1.0031 & - \\
\hline 1.50 & 1.0282 & 1.0517 & 1.0183 & & 1.0138 & 1.0430 & 1.0069 & 1.0367 & 1.0036 & 1.0320 \\
\hline 2 & 1.0497 & & 1.0323 & 1.1545 & 1.0243 & & 1.0122 & & 1.0064 & \\
\hline 3 & 1.1087 & & 1.0713 & 1.3224 & 1.0540 & & 1.0273 & & 1.0143 & \\
\hline 4 & 1.1863 & & 1.1237 & & 1.0941 & & 1.0481 & & 1.0253 & \\
\hline 5 & 1.2792 & & 1.1876 & & 1.1436 & & 1.0742 & & 1.0392 & \\
\hline
\end{tabular}

\section{Conclusions}

The concept of the CDF method applicable to beams presented in this paper is successfully applied to study the large amplitude free vibration behavior of tapered Timoshenko beams with axially immovable ends. The influence of the taper parameter on the linear and non-linear frequency parameter has been studied for two different tapered Timoshenko beam boundary conditions. Accurate closed form expressions for $\left(\frac{\omega_{N L}}{\omega_{L}}\right)^{2}$ for the hinged-hinged, clamped-clamped beam boundary conditions are obtained in terms of the maximum amplitude ratio, taper ratio and slenderness ratio for the assumed single term admissible function for the total rotation $\theta$.

\section{Acknowledgements}

The authors would like to thank the authorities of the University College of Engineering, Jawaharlal Nehru Technological University Kakinada (JNTUK), for sponsoring and presenting the research paper under TEQIP-II.

\section{Nomenclature}

$A$ - area of cross section

E - Young's modulus 


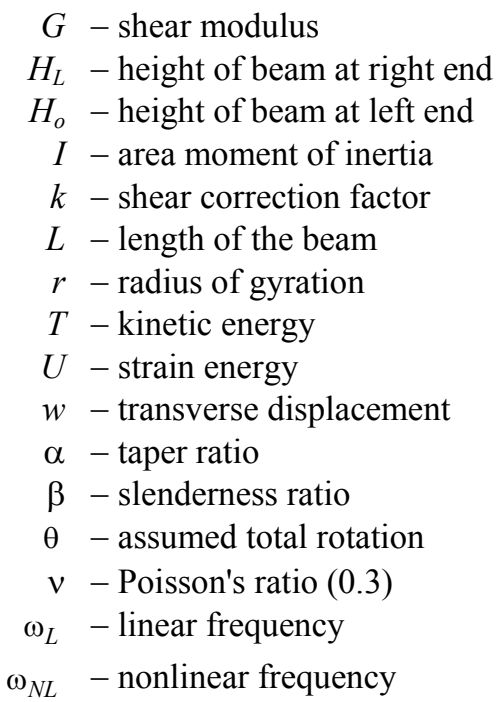

\section{References}

[1] Abrate S. (1995): Vibration of non-uniform rods and beams. - Journal of Sound and Vibration, vol.185, No.4, pp.703-716.

[2] Byoung Koo Lee, Jong Kook Lee and, Tae Eun Lee and Sun Gi Kim (2002): Free vibrations of tapered Beams with general boundary condition. - (KSCE) Journal of Civil Engineering, vol.6, No.3, pp.283-288.

[3] De Rosa M.A. and Auciello N.M. (1996): Free vibrations of tapered beams with flexible ends. - Journal of Computers and Structures, vol.60, No.2, pp.197-202.

[4] De Rosa M. A. and Lippiello M. (2009): Natural vibration frequencies of tapered beams. - Journal of Engineering Transactions, vol.57, No.1, pp.45-66.

[5] Clementi F., Demeio L., Mazzilli C.E.N. and Lenci S. (2015): Nonlinear vibrations of non-uniform beams by the MTS asymptotic expansion method. - Continuum Mech. Thermodyn., vol.27, pp.703-717.

[6] Firouz-Abadi R.D., Haddadpour H. and Novinzadeh A.B. (2007): An asymptotic solution to transverse free vibrations of variable-section beams. - Journal of Sound and Vibration, vol.304, pp.530-540.

[7] Zamorska I. (2010): Free transverse vibrations of non- uniform beams. - Scientific Research of the Institute of Mathematics and Computer Science, vol.9, No.2, pp.244-250.

[8] Jung Woo Lee (2016): Free vibration analysis using the transfer-matrix method on a tapered beam. - Journal of Computers and Structures, vol.164, pp.75-82.

[9] Raju L.S., Raju K. and Rao G.V. (1976): Large amplitude free vibrations of tapered beams. - AIAA Journal, vol.14, No.2, pp.280-282.

[10] Mahmoud A.A., Abdelghany S.M. and Ewis K.M. (2013): Free vibrations of uniform and non uniform Euler beam using differential transformation method. - Asian Journal of Mathematics and Applications, vol.2013, article id ama0097, pp.1-16.

[11] MeeraSaheb., Rao K.G.V. and Janardhan G.R. (2007): Free vibration analysis of Timoshenko beams using coupled displacement field method. - Journal of Structural Engineering, vol.34, pp.233-236.

[12] Mehmet Cem Ece., Aydogdu M. and Taskin V. (2007): Vibration of a variable cross-section beam. - Journal of Mechanics Research Communications, vol.34, pp.78-84.

[13] Minmao Liao and Hongzhi Zhong (2008): Non-linear vibration analysis of taper Timoshenko beams. - Chaos, Solitons and Fractals, vol.36, pp.1267-1272. 
[14] Mahmoud Bayat, Iman Pakar and Mahdi Bayat (2011): Analytical study on the vibration frequencies of tapered beams. - Latin Ameriacan Journal of Solids and Structures, vol.8. pp.149 -162

[15] Mohamed Hussien Taha and Samir Abohadima (2008): Mathematical model for vibrations of non-uniform flexural beams. - Journal of Engineering Mechanics, vol.15, No.1, pp.3-11.

[16] Lewandowski R. (1987): Application of the Ritz method to the analysis of non linear free vibrations of beams. Journal of Sound and Vibration, vol.114, pp.91-101.

[17] Ramazan A., Jafari-Talookolaei and Maryam Abedi (2014): An exact solution for the free vibration analysis of Timoshenko beams. - Review of Applied Physics, vol.3, pp.12-17.

[18] Rossi R.E. and Laura P.A.A. (1993): Numerical experiments on vibrating, linearly tapered Timoshenko beams. Journal of Sound and Vibration, vol.168, No.1, pp.179-183.

[19] Si Yuan, Ye K., Xiao C., Williams F.W. and Kennedy D. (2007): Exact dynamic stiffness method for nonuniform Timoshenko beam vibrations and Bernoulli-Euler column buckling. - Journal of Sound and Vibration, vol.303, pp.526-537.

[20] Kukla S. and Zamojska I. (2005): Application of the Green's function method in free vibration analysis of non uniform beams. - Scientific Research of the Institute of Mathematics and Computer Science, vol.4, No.1, pp.87-94.

[21] Hoseini S.H., Pirbodaghi T., Ahmadian M. T. and Farrahi G.H. (2009): The large amplitude free vibrations of tapered beams: an analytical approach. - Mechanics Research Communications, vol.36, pp.892-897.

[22] Zhou D. and Cheung Y.K. (2000): The free vibration of a type of tapered beams. - Journal of Comput. Methods Appl. Mech. Engrg, vol.188, pp.203-216.

Received: May 22, 2017

Revised: June 20, 2018 